\title{
Cuestionario de esquemas de Young CEY-S3: Propiedades psicométricas en una muestra chilena mixta
}

\section{Young Schema Questionnaire YSQ-S3: Psychometric properties in an adult mixed sample from Chile}

\author{
Álvaro, Quiñones \\ Instituto de Ciencias Humanas Aplicadas (ICHA), Facultad de Psicología, Universidad San Sebastián, Santiago, Chile. \\ Patricio, Ramírez \\ Facultad de Psicología, Universidad del Desarrollo, Chile. \\ Jordi, Cid \\ Grupo de Investigación de Salud Mental y Adicciones. Institut d’Investigació Biomèdica de Girona (IDIBGI) \\ Institut Assitència Sanitària. Girona, España. \\ Roberto, Melipillán \\ Facultad de Psicología, Universidad del Desarrollo, Chile. \\ Carla, Ugarte \\ Instituto de Ciencias Humanas Aplicadas (ICHA), Facultad de Psicología, Universidad San Sebastián, Santiago, Chile. \\ Ramón, Florenzano \\ Facultad de Psicología, Universidad del Desarrollo, Chile.
}

Rec (25 de julio de 2018) Acept (30 de octubre de 2018)

Agradecimientos: A los pacientes y estudiantes universitarios que participaron voluntariamente en la presente investigación.

A la Unidad de Trastornos de Personalidad del Policlínico de Psiquiatría del Hospital del Salvador, Santiago, Chile.

Al Servicio de Psiquiatría Leonor Mascayano, Hospital Clínico Regional Dr. Guillermo Grant Benavente, Concepción, Chile.

La presente investigación fue financiada por el Fondo interno UDD (n²3400059) cuyo investigador principal es Álvaro Quiñones, titulado: “Esquemas desadaptativos tempranos en psicología clínica y evaluación psicométrica” de la Dirección de Investigación, Universidad del Desarrollo, Chile. Los investigadores Carla Ugarte y Álvaro Quiñones agradecen a la Facultad de Psicología de la Universidad San Sebastián el permanente apoyo prestado para la presente investigación.

Álvaro Quiñones Bergeret y Jordi Cid Colom agradecen a Jeffrey Young por autorizar la utilización del Cuestionario de Esquemas de Young CEY-S3 en la presente investigación en Chile.

Correspondencia: Álvaro Quiñones, alvaroquinonesb@gmail.com, Universidad San Sebastián, Campus Los Leones Lota 2465, Providencia, Santiago, Chile. 


\title{
Resumen
}

El Cuestionario de Esquemas de Young CEY-S3 es un instrumento que mide una taxonomía de 18 temas psicológicos centrales denominados esquemas desadaptativos tempranos, los cuales subyacerían a los trastornos de personalidad y a otros trastornos mentales. Esta investigación pretende estudiar las propiedades psicométricas del CEY-S3 en población chilena. Una muestra mixta de 85 pacientes de dos unidades de psiquiatría en hospitales públicos con diagnóstico de al menos un trastorno de la personalidad, y de 207 estudiantes universitarios no consultantes de dos universidades privadas. Los resultados muestran un buen ajuste para el modelo de 18 factores, una buena capacidad de discriminación por sexo y muestra clínica y no clínica, así como la consistencia interna de 17 de las 18 escalas es adecuada $(\alpha>0.70)$. Estos resultados están en concordancia con los estudios de validación en distintas lenguas y culturas, lo que apoya su empleo en ámbitos clínicos o de investigación.

Palabras clave: Esquemas desadaptativos tempranos, Cuestionario de Esquemas de Young, Propiedades psicométricas

\begin{abstract}
Young Schema Questionnaire, CEY-S3, is an instrument that measures a taxonomy of 18 central psychological themes called early maladaptive schemas, which would underlie personality disorders and other mental disorders. The aim of this investigation is analyze psychometric properties of CEY-S3 in a Chilean mixed sample of 85 patients with a diagnosis of personality disorders from two psychiatric units in public hospitals and a 207 university students sample from two private universities. The results show a good fit for the 18 factor model, a good ability to discriminate by sex and clinical and non-clinical sample, as well as the internal consistency of 17 of the 18 scales is adequate $(\alpha>0.70)$. These results are in accordance with the validation studies in different languages and cultures, which supports their use in clinical or research fields.

Keywords: Early maladaptative schemas; Young Schema Questionnaire, psychometric properties.
\end{abstract}

\section{Introducción}

La Terapia de Esquemas (TE) es una terapia integrativa desarrollada para conceptualizar y tratar a las personas afectadas con trastornos de la personalidad que integra elementos de la terapia cognitivo-conductual de los enfoques psicodinámicos y humanistas (Young, Klosko \& Weishaar, 2003). La TE ha mostrado resultados satisfactorios en los estudios de efectividad para distintos trastornos de la personalidad (Masley, Gillanders, Simpson \& Morag, 2011; Jacob \& Arntz, 2013).

Uno de los constructos centrales de la TE en el que se vertebra la organización de la conceptualización del caso y el tratamiento, son los esquemas desadaptativos tempranos, que se definen como temas amplios y persistentes formados por recuerdos, emociones, pensamientos y sensaciones corporales que hacen referencia al análisis de uno mismo y de las relaciones con los demás, se originan en la infancia a través de la interacción entre las experiencias de la infancia, el temperamento y la cultura, y se van desarrollando a lo largo de la vida de las personas siendo disfuncionales o no facilitando la adaptación en la vida adulta. Se han definido 18 esquemas que surgen de las necesidades emocionales no cubiertas y que se han agrupado en dimensiones que representan un componente importante de las necesidades emocionales básicas de la infancia (Young \& Klosko, 2007), tal como podemos observar en la tabla 1 . 
Tabla 1. Dominios de Esquemas, Necesidades emocionales no cubiertas y Esquemas

\begin{tabular}{|c|c|c|c|}
\hline Dominio & Definición dominio & $\begin{array}{l}\text { Necesidades } \\
\text { emocionales no } \\
\text { cubiertas }\end{array}$ & Esquemas \\
\hline $\begin{array}{l}\text { Desconexión y } \\
\text { Rechazo }\end{array}$ & $\begin{array}{l}\text { Expectativas de no satisfacer las } \\
\text { propias necesidades de seguridad, } \\
\text { aceptación y respeto }\end{array}$ & $\begin{array}{l}\text { Vínculos seguros hacia } \\
\text { los demás (seguridad } \\
\text { básica, estabilidad, } \\
\text { previsibilidad, amor, } \\
\text { cuidado emocional, } \\
\text { aceptación y halagos). }\end{array}$ & $\begin{array}{l}\text { Privación Emocional, } \\
\text { Abandono, Desconfianza/ } \\
\text { Abuso, Imperfección/Vergüenza } \\
\text { Aislamiento social }\end{array}$ \\
\hline $\begin{array}{l}\text { Deterioro en la } \\
\text { Autonomía y } \\
\text { Rendimiento }\end{array}$ & $\begin{array}{l}\text { Expectativas que interfieren con } \\
\text { la propia habilidad de funcionar } \\
\text { independientemente o de } \\
\text { desempeñar con éxito }\end{array}$ & $\begin{array}{l}\text { Autonomía, competencia } \\
\text { y sentido de identidad }\end{array}$ & $\begin{array}{l}\text { Dependencia/Incompetencia, } \\
\text { Fracaso, Vulnerabilidad al } \\
\text { peligro y Apego. }\end{array}$ \\
\hline $\begin{array}{l}\text { Dirigido a las } \\
\text { necesidades de } \\
\text { los demás }\end{array}$ & $\begin{array}{l}\text { Excesivamente focalizados en los } \\
\text { deseos de los demás, en detrimento } \\
\text { de las propias necesidades }\end{array}$ & $\begin{array}{l}\text { La libertad de expresar } \\
\text { las necesidades y } \\
\text { emociones (válidas) }\end{array}$ & $\begin{array}{l}\text { Subyugación } \\
\text { Autosacrificio } \\
\text { Búsqueda de Aprobación }\end{array}$ \\
\hline $\begin{array}{l}\text { Deterioro en los } \\
\text { límites }\end{array}$ & $\begin{array}{l}\text { Una deficiencia en los límites } \\
\text { internos, en las responsabilidades } \\
\text { con los demás o en los objetivos a } \\
\text { largo plazo (Insuficiente Autocontrol } \\
\text { y Grandiosidad). }\end{array}$ & $\begin{array}{l}\text { Los límites realistas, } \\
\text { autocontrol y frustración }\end{array}$ & $\begin{array}{l}\text { Grandiosidad } \\
\text { Límites }\end{array}$ \\
\hline $\begin{array}{l}\text { Sobrevigilancia e } \\
\text { Inhibición }\end{array}$ & $\begin{array}{l}\text { Un excesivo énfasis en controlar los } \\
\text { propios sentimientos espontáneos } \\
\text { y conductas para evitar cometer } \\
\text { errores }\end{array}$ & La espontaneidad y juego & $\begin{array}{l}\text { Negatividad } \\
\text { Inhibición Emocional } \\
\text { Metas Inalcanzables }\end{array}$ \\
\hline
\end{tabular}

Uno de los aspectos relevantes y distintivos de la terapia de esquemas (Young, 1990), y concretamente de su creador Jeffrey Young, ha sido el desarrollo de cuestionarios que evalúan los distintos constructos que propone la terapia de esquemas (Cid, 2010). El cuestionario que más impacto ha tenido ha sido el cuestionario de Esquemas de Young, una medida de los esquemas desadaptativos tempranos. En este sentido, se puede considerar que su contribución a la investigación significó un antes y un después en el estudio del papel de los esquemas como factor de riesgo y/o vulnerabilidad en la psicopatología (Cid, 2016).

Existen distintas versiones del cuestionario de esquemas, que han ido cambiando en función de los cambios en el modelo teórico (Oei y Baranoff, 2007). El modelo original (Young, 1990) proponía una lista de 15 esquemas, y se construyeron dos versiones, una extensa de 205 ítems (CEY-L) y otra reducida de 75 ítems (CEY-VR). Posteriormente, con la revisión del modelo de Terapia de Esquemas (Young, Klosko \& Weishaar, 2003) donde se pasó de un modelo de 15 a 18 esquemas, se construyó el Cuestionario de Esquemas, CEY-S3, de 90 ítems que mide los 18 esquemas propuestos por el nuevo modelo (Young, 2006). Este cuestionario ha sido adaptado a distintas lenguas y culturas: rumano (Trip, 2006), Portugués (Rijo \& Gouveia, 2008), Finés (Saariaho, Saariaho, Karila, \& Joukamaa, 2009), Turco (Soygüt, Karaosmanoglu \& Cakir, 2009), Francés en Canadá (Hawke \& Provencher, 2012); Alemán (Kriston, Schäfer, Jacob, Härter \& Hölzel, 2013), Griego (Lyrakos, 2014), Danés (Bach, Simonsen, Christoffersen \& Kriston, 2015), Coreano (Lee, Choi, Rim, Won \& Lee, 2015), Polaco (Oettingen, Chodkiewicz, Mącik \& Gruszczyńska, 2017); Thai (Sakulsriprasert, Phukao, Kanjanawong \& Meemon, 2016); y finalmente al Español en España con estudiantes universitarios (Calvete, Orue, \& González-Diez, 2013) y con población clínica (Cid, 2016). Estos estudios de adaptación y validación psicométrica del CEY-S3 presentan unas buenas propiedades psicométricas, realizándose alguno de ellos en muestras grandes de más de 1000 sujetos (Bach, et al., 2015; Kriston, et al., 2013; Oettingen et al. (2017); Lyrakos (2014) y mayoritariamente en muestras con población no clínica, a excepción del Lyrakos (2014). Dos de ellos utilizan el análisis factorial exploratorio (Saritas \& Gençoz, 2011; Soygüt, et al., 2009) y otros análisis factorial confirmatorio (Bach, et al., 2015; Calvete, et al. 2013; Hawke et al., 2012; Kriston, 
et al., 2013; Lee et al., 2015; Lyrakos, 2014; Oettingen et al., 2017; Rijo et al., 2008; Saariaho, et al., 2009). En estos últimos estudios todos los índices CFA del análisis factorial confirmatorio muestran un ajuste aceptable para el modelo de 18 factores; $\chi 2$ normalizado por debajo de 3.00; CFI por encima de 0.90 , RMSEA por debajo de 0,50, SRMR por debajo de 0,80 . Hay tres excepciones que son los estudios en lengua alemana de Kriston et al., (2013), el danesa de Bach et al., (2015) y el de lengua polaca Oettingen et al., (2017), con problemas en índices chi-cuadrado de modelos, y en los índices CFI y CFA, que nos indican que el modelo no cumplía los requisitos de ajuste para el análisis de los 18 esquemas. En cambio, encuentran que cuando hacen un modelo general de esquemas correlacionado con los 18 esquemas específicos, los modelos de ajuste son correctos. Justifican estos hallazgos en el análisis factorial confirmatorio de ítems porque estos índices son muy sensibles cuando los tamaños de la muestra son mayores a 300 y son modelos complejos (Hair, Black, Babin \& Anderson, 2009).

Todos estos estudios encuentran unos índices de fiabilidad adecuados para cada una de las 18 escalas y uno de ellos encuentra una buena estabilidad test-retest (Calvete et al., 2013). En relación a la validez convergente, encuentran asociaciones positivas con índices de correlación entre mo- derados y altos, entre síntomas y los esquemas. En cuanto a la validez discriminante entre muestras clínicas y muestras de personas sanas, es adecuada, puntuando significativamente más elevadas las muestras clínicas, aspecto que se corrobora en todas las investigaciones, independientemente de la versión del cuestionario (Cid, 2016) y de la patología que se compare con las muestras no clínicas de personas sanas; ocurre en la depresión (Hirsch, Hautekeete \& Kochman, 2001; Shah \& Waller, 2000; Waller, Shah, Ohanian \& Elliott, 2001), en la ansiedad y en los trastornos de la personalidad (Lee, Taylor \& Dunn, 1999; Bach, et al. 2015 y Kriston, et al. 2013; Cid, 2016).

Por último, cabe destacar que los esquemas se comportan como rasgos de personalidad, aunque algunos de ellos podrían comportarse como estado-dependientes (Riso, Froman, Raouf, Gable, Maddux, Turini-Santorelli, et al., 2006); y aportan validez incremental al estudio de la personalidad y sus trastornos (Thimm, 2011; Cid, 2016).

El objetivo del presente estudio es presentar las propiedades psicométricas: fiabilidad, estructura factorial y capacidad de discriminación, de la adaptación del Cuestionario de Esquemas de Young de 90 ítems que mide 18 esquemas propuestos en población chilena adulta.

Tabla 2. Escalas del CEY-S3 y descripción breve de los 18 Esquemas

\begin{tabular}{ll}
\hline Escalas & Descripción breve de los Esquemas \\
\hline Privación Emocional & $\begin{array}{l}\text { La expectativa que no se van a satisfacer adecuadamente con los otros las propias } \\
\text { necesidades emocionales }\end{array}$ \\
\hline Abandono & $\begin{array}{l}\text { La expectativa que perderán a las personas significativas o les dejarán emocionalmente o } \\
\text { físicamente }\end{array}$ \\
\hline Desconfianza & La expectativa que los demás les herirán, abusarán o humillarán \\
\hline Imperfección & $\begin{array}{l}\text { Avergonzado/inútil como consecuencia de los sentimientos de ser malo, inferior o } \\
\text { desvalido }\end{array}$ \\
\hline Aislamiento social & Sentimiento de ser diferente de los demás, de estar fuera de los grupos \\
\hline Dependencia & Creencia de que uno es incapaz de realizar las tareas cotidianas sin ayuda de los demás \\
\hline Fracaso & $\begin{array}{l}\text { Creencia que es un fracaso en la escuela o en su carrera profesional y que inevitablemente } \\
\text { fallará en la vida }\end{array}$ \\
\hline Vulnerabilidad al peligro & Miedo exagerado a que ocurran malas cosas, que no se pueden prevenir o hacer frente \\
\hline Apego & Implicación excesiva, y constante búsqueda de soporte de las personas significativas \\
\hline Subyugación & Conformidad con los demás con el fin de evitar consecuencias temidas \\
\hline Auto-sacrificio & De forma voluntaria centrado en las necesidades de los demás en vez de en las propias \\
\hline Inhibición Emocional & Inhibición de la expresión de las emociones y de la espontaneidad \\
\hline Metas inalcanzables & Estándares personales altos en productividad, rendimiento y conducta \\
\hline Grandiosidad & Creencia que uno es superior a los demás, lo que le da derechos o privilegios especiales \\
\hline Insuficiente autocontrol & Dificultades con el propio autocontrol, la perseverancia y la demora de la gratificación \\
\hline
\end{tabular}




\begin{tabular}{ll}
\hline Escalas & Descripción breve de los Esquemas \\
\hline Búsqueda de aprobación & $\begin{array}{l}\text { La propia validez y autoestima depende de la aprobación o de obtener la atención de los } \\
\text { demás }\end{array}$ \\
\hline Castigo & $\begin{array}{l}\text { La creencia y expectativa que las personas serán duramente castigadas cuando cometan } \\
\text { errores }\end{array}$ \\
\hline Pesimismo & Expectativas que todas las cosas saldrán mal \\
\hline
\end{tabular}

\section{Método}

\section{Participantes}

Una muestra mixta, constituida por pacientes atendidos en unidades de psiquiatría y de estudiantes universitarios. El total de la muestra fue de 292 voluntarios, 85 corresponden a muestra clínica y 207 a estudiantes. Todos respondieron al CEY-S3, 66 (22,6 \%) hombres y 226 (77,4\%) mujeres. Las edades estaban comprendidas entre los 18 y 70 años, con una media de edad de 26,30 ( $\mathrm{DE}=11,53)$. En lo que concierne al estado civil, 219 (75\%) participantes eran solteros, $52(17,80 \%)$ estaban casados o eran pareja de hecho, $18(6,16 \%)$ estaban separados o divorciados y 1 personas eran viudos/as $(0,04 \%)$. Un 83,23\% $(n=243)$ de la muestra tiene un nivel educativo alto (estudios universitarios), un $10,95 \%(\mathrm{n}=32)$ un nivel educativo medio (pre-universitario), un $5,82 \%(\mathrm{n}=17)$ un nivel educativo bajo. La muestra de voluntarios estudiantes está compuesta por 207 estudiantes universitarios (78\% mujeres, con una edad media de 21,06 años, rango 18 a 48 años) y la muestra clínica está compuesta por 85 personas correspondientes a la Unidad de Trastornos de Personalidad del Policlínico de Psiquiatría del Hospital del Salvador y al Servicio de Psiquiatría Leonor Mascayano, Hospital Clínico Regional Dr. Guillermo Grant Benavente de Concepción (79,8\% mujeres, con una edad media de 39,40 años, rango de 18 a 70 años). La muestra clínica está compuesta por personas que recibieron todos ellos un diagnóstico de Trastorno de la Personalidad, 7,05\% $(\mathrm{n}=6)$ de Cluster A (trastorno de personalidad esquizoide, esquizotípico o paranoide), $62,35 \%(\mathrm{n}=53)$ de Cluster B (trastorno de personalidad límite, histriónico o narcisista), un $14,12 \%(n=12)$ de Cluster $\mathrm{C}$ (trastorno de personalidad por evitación, por dependencia u obsesivo-compulsivo) y $14,12 \%(\mathrm{n}=12)$ trastorno de personalidad no especificado. De éstos, presentan comorbilidad un 56,7\% $(\mathrm{n}=48)$, pues recibieron un diagnóstico de Trastornos mentales del Eje I, principalmente trastornos depresivos $(\mathrm{n}=31)$, trastornos adaptativos $(\mathrm{n}=10)$ y otros trastornos como adicciones, ansiedad y de conducta alimentaria $(n=7)$.

\section{Instrumento}

Cuestionario de Esquemas de Young-Versión Tercera Reducida (CEY-S3 Young, 2006) es un inventario autoadminis- trado de 90 ítems desarrollado para medir una taxonomía de 18 Esquemas, denominados Esquemas Desadaptativos Tempranos (EDT). La adaptación española del Cuestionario de Esquemas de Young en su versión reducida de 90 ítems la han realizado Cid y Torrubia (2010) con la autorización pertinente del autor quien tiene la propiedad intelectual de los Cuestionarios de Esquemas de Young (Young, 1994; 1999; 2006). El procedimiento de adaptación del CEY fue la traducción del inglés al español, retrotraducción y la administración a una pequeña muestra para delimitar la comprensión, todo ello previo a la administración a una muestra para delimitar las adecuadas propiedades psicométricas (Cid 2016). Cada ítem se puntúa utilizando una escala de 6 puntos [ $1=$ no me describe, $2=$ la mayoría de veces falso, $3=$ más verdadero que falso, $4=$ en ocasiones verdadero, $5=$ la mayoría de veces verdadero, $6=$ me describe perfectamente]. Se realizó una adaptación idiomática del CEY-S3 a las expresiones del español de Chile y a la cultura chilena, a partir del CEY-S3 y del original en inglés por parte de dos profesionales de la salud, bilingües español e inglés.

\section{Procedimiento}

La muestra fue no probabilística e incluyó dos unidades de psiquiatría en hospitales públicos y dos universidades privadas. La muestra de personas no consultantes está compuesta por estudiantes de psicología de dos universidades con sede en sur de Chile, quienes respondieron el CEY-S3 a través de un formulario online.

Para el acceso a la muestra clínica se contactó a los directores de los Servicios de Psiquiatría del Hospital del Salvador y del Hospital Clínico Regional Dr. Guillermo Grant Benavente, a quienes se les presentó el proyecto para su análisis, aprobación, acceso a los pacientes y a su ficha clínica con los resguardos de confidencialidad. Posterior a la aprobación del proyecto, se contrató a dos psicólogos en Santiago y dos en Concepción para administrar los instrumentos a la muestra de manera presencial. Por otra parte, los diagnósticos clínicos de la muestra fueron obtenidos por los investigadores responsables a través del acceso a la ficha clínica.

En último lugar, tanto para la aplicación del Cuestionario de Esquemas de Young-Versión Tercera Reducida (CEY-S3; Young, 2006) a los estudiantes como a los pacientes, se procedió a la firma de un consentimiento informado. Los 
comités de ética que visan los estudios con pacientes en los dos servicios clínicos mencionados entregaron su aprobación para encuestar a los pacientes. De la misma manera, las respectivas universidades visaron el proyecto.

\section{Análisis de datos}

Para estudiar la validez de constructo del CEY-S3 se procedió a realizar un análisis factorial con el método de estimación de los parámetros del modelo, un método recomendado cuando se analiza ítems de escalas de tipo ordinal. Por otro lado, se estudió la confiabilidad del CEY-S3 en términos de su consistencia interna, a través de los índices alpha de Cronbach y las correlaciones totales sin el ítem. En lo que respecta a la capacidad discriminativa de los ítems del CEY-S3, se analizó en función de las siguientes variables: sexo (hombre y mujer) y tipo de muestra (estudiantes versus pacientes atendidos en unidades de psiquiatría) a través de análisis de la varianza t-test. Por último, los análisis de datos se realizaron con los paquetes estadísticos WLSMV en Mplus 7.2 (análisis factoriales) y SPSS 20 (descriptivos y comparaciones t-test).

\section{Fiabilidad}

La capacidad de discriminación de los ítems medida con las correlaciones elemento total corregido excede del punto de corte recomendado de 0,40 en todos los ítems, a excepción de tres ítems de la escala de Grandiosidad y dos ítems, uno para la escala de Aislamiento Social, otro para Vulnerabilidad al Peligro y otro para Apego. La consistencia interna de 17 de las 18 escalas es adecuada, $\alpha$ Cronbach's $>0.70$, a excepción de la escala de Grandiosidad, con un índice de 0,64 .

Tabla 3. Correlaciones elemento total corregido e Índices de Fiabilidad

\begin{tabular}{lcccccc}
\hline & Ítem 1 & Ítem 2 & Ítem 3 & Ítem 4 & Ítem 5 & $\begin{array}{c}\text { Alpha } \\
\text { Cronbach }\end{array}$ \\
\hline Privación Emocional & .687 & .638 & .544 & .753 & .636 & 0.84 \\
Abandono & .701 & .713 & .827 & .704 & .626 & 0.88 \\
Imperfección & .680 & .688 & .452 & .669 & .607 & 0.82 \\
Desconfianza & .551 & .671 & .642 & .653 & .619 & 0.83 \\
Aislamiento Social & .658 & .361 & .544 & .728 & .703 & 0.81 \\
Fracaso & .675 & .618 & .777 & .757 & .780 & 0.88 \\
Dependencia & .569 & .418 & .460 & .528 & .595 & 0.74 \\
Vulnerabilidad Peligro & .604 & .653 & .492 & .316 & .565 & 0.76 \\
Apego & .528 & .604 & .392 & .651 & .577 & 0.77 \\
Autosacrificio & .513 & .581 & .588 & .499 & .688 & 0.79 \\
Subyugación & .421 & .606 & .505 & .631 & .562 & 0.77 \\
Inhibición Emocional & .495 & .573 & .478 & .560 & .538 & 0.76 \\
Metas inalcanzables & .474 & .605 & .424 & .488 & .497 & 0.74 \\
Insuficiente Autocontrol & .460 & .544 & .426 & .507 & .455 & 0.72 \\
Grandiosidad & .357 & .449 & .484 & .358 & .340 & 0.64 \\
Admiración/ & .518 & .615 & .564 & .660 & .625 & 0.83 \\
Búsqueda Aprobación & .639 & .608 & .611 & .489 & .503 & 0.79 \\
Pesimismo & .595 & .429 & .687 & .652 & .461 & 0.78 \\
Castigo & & & & & & \\
\hline
\end{tabular}




\section{Estructura Factorial CEY-S3}

Los resultados del análisis factorial se muestran en la Tabla 4, con los pesos de los factores (pesos estandarizados de la regresión) para cada ítem. Todos los ítems tienen una saturación en el factor superior al límite de 0.40 , a excepción de uno de Insuficiente Autocontrol.

Los resultados muestran un modelo de ajuste razonable para el modelo de 18 factores de primer orden $\left[\chi^{2}=\right.$
$5074,796 \mathrm{df}=3762 \mathrm{p}<.0000 ; \mathrm{TLI}=0,931 ; \mathrm{CFI}=0,935 ; \mathrm{RM}-$ $\mathrm{SEA}=0,035,90 \%$ CI: de 0.032 a 0.037$]$. El test de discrepancia chi-cuadrado muestra un buen ajuste, con los índices CFI y TLI que están por encima del 0,90 recomendado y un RMSEA menor a los valores recomendados de 0,50. Los valores residuales del WRMR $=1.171$ (Weighted Root Mean Square Residual) son superiores al valor recomendado de 1.000 .

Tabla 4. Análisis Factorial Confirmatorio de los items

\begin{tabular}{lccccc}
\hline & Ítem 1 & Ítem 2 & Ítem 3 & Ítem 4 & Ítem 5 \\
\hline Privación Emocional & 0.477 & 0.719 & 0.619 & 0.804 & 0.795 \\
Abandono & 0.851 & 0.780 & 0.733 & 0.929 & 0.729 \\
Imperfección & 0.750 & 0.459 & 0.768 & 0.863 & 0.869 \\
Desconfianza & 0.709 & 0.833 & 0.879 & 0.816 & 0.871 \\
Aislamiento Social & 0.673 & 0.786 & 0.841 & 0.754 & 0.752 \\
Fracaso & 0.770 & 0.811 & 0.726 & 0.871 & 0.777 \\
Dependencia & 0.794 & 0.795 & 0.869 & 0.843 & 0.885 \\
Vulnerabilidad Peligro & 0.695 & 0.553 & 0.577 & 0.787 & 0.871 \\
Apego & 0.740 & 0.698 & 0.745 & 0.542 & 0.739 \\
Autosacrificio & 0.665 & 0.775 & 0.613 & 0.805 & 0.704 \\
Subyugación & 0.537 & 0.600 & 0.686 & 0.777 & 0.975 \\
Inhibición Emocional & 0.466 & 0.727 & 0.945 & 0.405 & 0.787 \\
Metas inalcanzables & 0.737 & 0.744 & 0.89 & 0.596 & 0.568 \\
Insuficiente Autocontrol & 0.655 & 0.507 & 0.474 & 0.392 & 0.725 \\
Grandiosidad & 0.570 & 0.649 & 0.414 & 0.920 & 0.543 \\
Admiración/Búsqueda Aprobación & 0.450 & 0.749 & 0.608 & 0.598 & 0.685 \\
Pesimismo & 0.530 & 0.831 & 0.888 & 0.716 & 0.64 \\
Castigo & 0.802 & 0.713 & 0.786 & 0.725 & 0.693 \\
\hline
\end{tabular}

Todos los factores están positivamente asociados con otros tal como muestra matriz de correlaciones entre escalas, con un rango entre, de 0,26 entre Pesimismo y Metas Inalcanzables y 0,99 entre Castigo y Apego. De estos factores, hay más de un $30 \%$ de asociaciones de esquemas superiores a .70, con las escalas de Castigo y Vulnerabilidad al Peligro como las que tienen correlaciones más altas con el resto de esquemas.

\section{Validez Discriminante}

Las comparaciones de medias t-test nos indican que en el esquema de Grandiosidad las mujeres puntúan significativamente más elevado que los hombres (Tabla 5). En cambio, en el resto de esquemas y en la puntuación total del Cuestionario de Esquemas CEY-S3 no se observan diferencias estadísticamente significativas entre las puntuaciones en función del sexo, aunque en tres esquemas Privación Emocional, Aislamiento Social y Autosacrificio se observa una tendencia a la significación. 
Tabla 5. Matriz de correlaciones entre las escalas del CEY-S3

\begin{tabular}{|c|c|c|c|c|c|c|c|c|c|c|c|c|c|c|c|c|c|c|}
\hline & 1 & 2 & 3 & 4 & 5 & 6 & 7 & 8 & 9 & 10 & 11 & 12 & 13 & 14 & 15 & 16 & 17 & 18 \\
\hline 1. Privación Emocional & 1 & - & - & - & - & - & - & - & - & - & - & - & - & - & - & - & - & - \\
\hline 2. Abandono & 0.51 & 1 & - & - & - & - & - & - & - & - & - & - & - & - & - & - & - & - \\
\hline 3. Imperfección & 0.52 & 0.78 & 1 & - & - & - & - & - & - & - & - & - & - & - & - & - & - & - \\
\hline 4. Desconfianza & 0.56 & 0.54 & 0.49 & 1 & - & - & - & - & - & - & - & - & - & - & - & - & - & - \\
\hline 5. Aislamiento Social & 0.58 & 0.70 & 0.75 & 0.72 & 1 & - & - & - & - & - & - & - & - & - & - & - & - & - \\
\hline 6. Fracaso & 0.65 & 0.76 & 0.79 & 0.67 & 0.73 & 1 & - & - & - & - & - & - & - & - & - & - & - & - \\
\hline 7. Dependencia & 0.55 & 0.63 & 0.61 & 0.56 & 0.54 & 0.79 & 1 & - & - & - & - & - & - & - & - & - & - & - \\
\hline 8. Vulnerabilidad Peligro & 0.64 & 0.62 & 0.64 & 0.66 & 0.69 & 0.78 & 0.81 & 1 & - & - & - & - & - & - & - & - & - & - \\
\hline 9. Apego & 0.63 & 0.65 & 0.53 & 0.74 & 0.80 & 0.62 & 0.61 & 0.76 & 1 & - & - & - & - & - & - & - & - & - \\
\hline 10.Autosacrificio & 0.66 & 0.65 & 0.68 & 0.76 & 0.73 & 0.73 & 0.64 & 0.79 & 0.73 & 1 & - & - & - & - & - & - & - & - \\
\hline 11. Subyugación & 0.49 & 0.49 & 0.44 & 0.64 & 0.65 & 0.56 & 0.47 & 0.70 & 0.68 & 0.83 & 1 & - & - & - & - & - & - & - \\
\hline 12.Inhibición Emocional & 0.45 & 0.45 & 0.32 & 0.57 & 0.45 & 0.32 & 0.39 & 0.38 & 0.51 & 0.58 & 0.43 & 1 & - & - & - & - & - & - \\
\hline 13.Metas inalcanzables & 0.34 & 0.60 & 0.73 & 0.39 & 0.61 & 0.66 & 0.48 & 0.53 & 0.48 & 0.60 & 0.35 & 0.38 & 1 & - & - & - & - & - \\
\hline 14.Insuficiente Autocontrol & 0.50 & 0.41 & 0.46 & 0.51 & 0.72 & 0.47 & 0.39 & 0.53 & 0.60 & 0.52 & 0.55 & 0.36 & 0.40 & 1 & - & - & - & - \\
\hline 15.Grandiosidad & 0.59 & 0.36 & 0.40 & 0.55 & 0.55 & 0.34 & 0.36 & 0.28 & 0.54 & 0.47 & 0.47 & 0.48 & 0.39 & 0.76 & 1 & - & - & - \\
\hline 16.Admiración/B Aprobación & 0.61 & 0.32 & 0.62 & 0.62 & 0.69 & 0.73 & 0.79 & 0.86 & 0.68 & 0.76 & 0.61 & 0.36 & 0.48 & 0.76 & 0.38 & 1 & - & - \\
\hline 17.Pesimismo & 0.54 & 0.38 & 0.33 & 0.67 & 0.58 & 0.42 & 0.41 & 0.51 & 0.62 & 0.59 & 0.56 & 0.32 & 0.26 & 0.79 & 0.58 & 0.69 & 1 & - \\
\hline 18. Castigo & 0.73 & 0.62 & 0.56 & 0.72 & 0.84 & 0.72 & 0.70 & 0.72 & 0.99 & 0.74 & 0.67 & 0.54 & 0.44 & 0.65 & 0.58 & 0.74 & 0.72 & 1 \\
\hline
\end{tabular}

Tabla 6. Comparaciones de medias t-test en función del sexo

\begin{tabular}{|c|c|c|c|c|c|c|}
\hline & & & $\begin{array}{c}\text { Hombres } \\
(\mathrm{n}=65)\end{array}$ & $\begin{array}{c}\text { Mujeres } \\
(\mathrm{n}=226)\end{array}$ & & \\
\hline & Media & DE & Media & DE & $t(n=289)$ & Sig. \\
\hline Privación Emocional & 12.15 & 6.46 & 10.65 & 6.18 & 1.71 & 0.09 \\
\hline Abandono & 13.54 & 6.75 & 14.26 & 7.09 & -0.73 & 0.46 \\
\hline Imperfección & 10.29 & 5.16 & 9.73 & 5.66 & 0.72 & 0.47 \\
\hline Desconfianza & 12.22 & 5.91 & 12.19 & 6.03 & 0.03 & 0.98 \\
\hline Aislamiento Social & 15.58 & 6.55 & 13.92 & 6.24 & 1.87 & 0.06 \\
\hline Fracaso & 11.02 & 6.73 & 11.50 & 6.21 & -0.54 & 0.59 \\
\hline Dependencia & 9.69 & 4.56 & 10.09 & 5.09 & -0.57 & 0.57 \\
\hline Vulnerabilidad Peligro & 11.42 & 5.82 & 12.04 & 6.06 & -0.74 & 0.46 \\
\hline Apego & 10.03 & 5.25 & 10.61 & 5.76 & -0.73 & 0.46 \\
\hline Autosacrificio & 16.42 & 5.91 & 17.93 & 5.95 & -1.81 & 0.07 \\
\hline Subyugación & 11.14 & 4.76 & 11.98 & 5.82 & -1.06 & 0.29 \\
\hline Inhibición Emocional & 13.98 & 5.63 & 12.58 & 5.95 & 1.70 & 0.09 \\
\hline Metas inalcanzables & 16.52 & 5.48 & 17.00 & 5.82 & -0.59 & 0.56 \\
\hline
\end{tabular}




\begin{tabular}{lcccccc} 
& Media & DE & Media & DE & t (n=289) & Sig. \\
Insuficiente Autocontrol & 13.85 & 6.01 & 13.89 & 5.40 & -0.05 & 0.96 \\
Grandiosidad & 13.75 & 4.72 & 15.19 & 5.12 & -2.03 & 0.04 \\
Admiración/ & 13.35 & 5.56 & 13.77 & 6.20 & -0.49 & 0.63 \\
Búsqueda Aprobación & 12.18 & 5.85 & 13.23 & 6.02 & -1.24 & 0.22 \\
Pesimismo & 13.11 & 5.65 & 12.37 & 5.57 & 0.94 & 0.35 \\
Castigo & 232.48 & 75.02 & 230.25 & 65.84 & -0.22 & 0.83 \\
Puntuación Total CEY & & & & & & \\
\hline
\end{tabular}

Se compararon las dos muestras de adultos estudiantes sanos y la muestra clínica compuesta por personas con un diagnóstico de Trastorno de la Personalidad, y se encontraron diferencias significativas entre los dos grupos para cada uno de los esquemas y para la puntuación total del
Cuestionario de Esquemas CEY-S3 $(p<0.001)$. La muestra clínica con diagnóstico de Trastorno de la personalidad obtuvo puntuaciones medias significativamente más elevadas que el grupo de adultos que eran estudiantes universitarios.

Tabla 7. Comparaciones de medias t-test de los esquemas en función del tipo de muestra, estudiantes versus clínica.

\begin{tabular}{|c|c|c|c|c|c|c|c|}
\hline & \multicolumn{2}{|c|}{$\begin{array}{l}\text { Clínica } \\
(\mathrm{n}=84)\end{array}$} & \multicolumn{2}{|c|}{$\begin{array}{c}\text { Estudiante } \\
(\mathbf{n}=\mathbf{2 0 7})\end{array}$} & \multirow[b]{2}{*}{$\mathbf{t}$} & \multirow[b]{2}{*}{ gl } & \multirow[b]{2}{*}{ Sig. } \\
\hline & Media & DE & Media & $\overline{D E}$ & & & \\
\hline Privación Emocional & 15.87 & 6.30 & 9.00 & 5.06 & 8.890 & 128.509 & .000 \\
\hline Abandono & 19.27 & 6.51 & 12.03 & 6.09 & 8.967 & 288 & .000 \\
\hline Imperfección & 13.50 & 6.12 & 8.38 & 4.55 & 6.934 & 121.914 & .000 \\
\hline Desconfianza & 16.77 & 5.70 & 10.34 & 5.04 & 9.489 & 289 & .000 \\
\hline Aislamiento Social & 17.72 & 6.46 & 12.92 & 5.75 & 6.203 & 288 & .000 \\
\hline Fracaso & 15.60 & 6.98 & 9.69 & 5.14 & 7.024 & 121.209 & .000 \\
\hline Dependencia & 14.41 & 5.90 & 8.24 & 3.15 & 9.034 & 101.260 & .000 \\
\hline Vulnerabilidad Peligro & 17.42 & 6.21 & 9.66 & 4.22 & 10.510 & 115.397 & .000 \\
\hline Apego & 15.09 & 6.21 & 8.68 & 4.22 & 8.540 & 110.065 & .000 \\
\hline Autosacrificio & 20.40 & 6.22 & 16.44 & 5.47 & 5.375 & 288 & .000 \\
\hline Subyugación & 16.33 & 5.90 & 9.95 & 4.28 & 9.000 & 120.052 & .000 \\
\hline Inhibición Emocional & 14.92 & 5.92 & 12.07 & 5.70 & 3.821 & 289 & .000 \\
\hline Metas inalcanzables & 19.07 & 5.05 & 16.01 & 5.77 & 4.244 & 289 & .000 \\
\hline Insuficiente Autocontrol & 17.73 & 5.41 & 12.31 & 4.77 & 8.433 & 288 & .000 \\
\hline Grandiosidad & 17.46 & 4.65 & 13.82 & 4.85 & 5.886 & 289 & .000 \\
\hline Admiración/Búsqueda Aprobación & 17.30 & 6.20 & 12.21 & 5.35 & 7.019 & 289 & .000 \\
\hline Pesimismo & 18.04 & 5.41 & 10.95 & 4.92 & 10.821 & 289 & .000 \\
\hline Castigo & 16.10 & 5.03 & 11.09 & 5.15 & 7.565 & 289 & .000 \\
\hline Puntuación Total CEY & 301.83 & 60.64 & 203.63 & 56.72 & 13.117 & 289 & .000 \\
\hline
\end{tabular}




\section{Discusión}

Este estudio presenta las propiedades psicométricas del Cuestionario de Esquemas de Young, CEY-S3, en muestras chilenas. Las propiedades psicométricas estudiadas nos indican una adecuada consistencia interna con índices de fiabilidad, similares a las obtenidas en los otros estudios de validación del CEY-S3. En nuestro estudio encontramos que la escala Grandiosidad tenía un índice alpha de Cronbach de 0,64 , al igual que ocurre en las adaptaciones de cuestionario al francés de Canadá con un alpha de Cronbach de 0,57 (Hawke et al., 2012), en la versión alemana con un índice de 0,67 (Kriston, et al., 2013), coreana con un 0,59 (Lee, et al., 2015) y polaca de 0,62 (Oettingen et al., 2017). En este sentido, cabe indicar que el esquema de Grandiosidad hace referencia a la creencia de que uno es superior a los demás, que le da derecho a privilegios y derechos especiales, y que no tiene que limitase por las reglas de reciprocidad que guían la interacción social habitual, donde los ítems del cuestionario en su versión reducida recogen sobre todo aspectos relacionados con "ser superior y especial" y donde es posible que las diferencias culturales expliquen este resultado.

Encontramos una estructura factorial coherente con el modelo teórico, dado que tanto el test de discrepancia chi-cuadrado y los índices global de ajuste, CFI, TFI y RMSEA muestran una buena bondad de ajuste del modelo de 18 factores. No obstante, en el presente estudio en los distintos ítems de cada una de las escalas que miden esquemas encontramos altas correlaciones entre ellas, sobre todo entre el esquema Apego y Castigo con un índice cerca de uno. Estas altas asociaciones entre factores conforman un aspecto que se observa en todas las adaptaciones a los diferentes países (Bach et al., 2015; Calvete et al., 2013; Hawke \& Provencher, 2012; Kriston et al., 2013; Rijo \& Gouveia, 2008; Saariaho et al., 2009). A nuestro modo de ver, estas altas asociaciones tienen que ver con naturaleza del constructo esquema y su evaluación. La identificación de los esquemas relacionados con el autoconcepto que se comportarían como rasgos de personalidad en la práctica clínica y en la evaluación psicométrica son métodos distintos. Los matices que aporta cada esquema en relación las dimensiones propuestas de desconexión y rechazo, deterioro en la autonomía y rendimiento, sobrevigilancia e inhibición, deterioro en los límites, dirigido a las necesidades de los demás son claros desde la perspectiva clínica de atribución de significados que dan coherencia a la visión del sí mismo, ya que podemos observar los pensamientos, los recuerdos asociados, las emociones, las atribuciones que se realizan. No obstante, en la evaluación mediante cuestionarios y cuando utilizamos las técnicas psicométricas para intentar validar estos constructos nos encontramos que los análi- sis que tenemos disponibles nos indican que esos matices que los esquemas aportan son difíciles de corroborar en los estudios de investigación. Las altas asociaciones que muestran los análisis factoriales entre esquemas que encontramos, como por ejemplo Apego y Castigo, desde la perspectiva psicométrica sería necesario fusionar ambas escalas y realizar un análisis con 17 factores, resultando una decisión difícil de sustentar desde la perspectiva clínica. Por este motivo, se buscan modelos de análisis factoriales confirmatorios donde tendríamos un modelo bifactorial, con un factor general que está correlacionado con el resto de factores (Bach et al., 2015; Kriston et al., 2013; Oettingen et al., 2017). Estos modelos complejos necesitan tamaños muestrales superiores a 1000 personas, siendo ésta una de las razones por la cual no hemos realizado un análisis de segundo orden de los dominios de esquemas. La otra razón es que en estos momentos existe una discusión en relación al número de dimensiones que agruparían a los esquemas. Los resultados de los análisis de segundo orden de las distintas versiones del CEY no apoyarían las cinco dimensiones de agrupación de los esquemas propuestas por el modelo teórico original (Cid, 2016) y en cambio sí un modelo de cuatro dimensiones que serían Desconexión y Rechazo, Deterioro en la Autonomía y Rendimiento, Excesiva Responsabilidad y Metas, Deterioro en los Límites (Bach, Lockwood \& Young, 2017).

A nivel de validez discriminante, observamos que el esquema de Grandiosidad muestra puntuaciones más elevadas en las mujeres que en los hombres, aspecto que debe interpretarse en función del tipo de muestra (Sines, Waller, Meyer \& Wigley, 2008) y buscando los aspectos culturales que pueden explicarlo. En nuestro estudio y a nuestro modo de ver, se podría relacionar con el alto nivel educativo, aunque es una hipótesis que debe ser estudiada. Los estudios de validación y adaptación que encontramos de los cuestionarios de esquemas obvian la comparación de sexos en sus análisis, siendo pocos los estudios donde se realizan comparaciones entre hombres y mujeres, apareciendo que las mujeres puntuarían más alto que los hombres en los esquemas (Welburn et al., 2002), pero no hay consistencia en qué esquemas puntúan más alto (Cid, 2016). Shorey, Anderson y Stuart (2012) encuentran que las mujeres que buscan tratamiento para el trastorno por dependencia al alcohol, puntúan más alto que los hombres a excepción de Grandiosidad. Estas diferencias entre la puntuación de esquemas entre hombres y mujeres se encuentran también en una muestra de trastorno por dependencia a opióides (Shorey, Stuart \& Anderson, 2012).

A nivel de validez discriminante, observamos diferencias significativas entre la población clínica con presencia de trastorno de personalidad y la población de estudiantes no clínica para la mayoría de los 18 esquemas y la puntua- 
ción total del cuestionario de esquemas CEY-S3.

Estos resultados son concordantes con los de Lee et al. (1999) donde las puntuaciones de las personas con trastornos de la personalidad eran más elevadas. Mauchanda, Lachenal-Chevallet y Cottraux (2011) comparan una muestra de controles sanos con pacientes con Trastorno de Personalidad Límite y encuentran puntuaciones mayores en estos últimos en todos los esquemas. Por último, destacar el estudio de Bach et al., (2015) donde encuentra diferencias significativas a nivel de puntuaciones de esquema entre un grupo de pacientes con Trastorno de Personalidad Límite, un grupo clínico general y un grupo no clínico, siendo más altas en el grupo de Trastorno de Personalidad Límite. Este aspecto es importante de tener en cuenta, desde la perspectiva de la adaptación cultural, ya que el cuestionario de esquemas CEY-S3 es capaz de discriminar e identificar los esquemas subyacentes a la patología de la personalidad en muchas culturas, aspecto muy interesante ya que nos permite su aplicación en la práctica clínica para evaluar personas afectadas con trastornos de la personalidad.

En resumen, la adaptación a la población chilena del CEY-S3 evalúa los 18 esquemas que propone el modelo está en concordancia con los estudios de validación a distintas lenguas y culturas obteniendo unas buenas cualidades psicométricas de consistencia interna, y en la estructura factorial de primer orden de 18 factores. No obstante, con esta versión existen menos datos disponibles de estabilidad temporal y se desconoce su comportamiento en otras muestras clínicas y no clínicas.

Limitaciones: no se aportan datos de estabilidad test-retest y tampoco de validez convergente, aunque se disponen de datos de esta validez.

\section{Referencias}

Bach, B., Simonsen, E., Christoffersen, P. \& Kriston, L. (2015). The Young Schema Questionnaire 3 Short Form (YSQ-S3): Psychometric properties and association with personality disorders in a Danish mixed sample. European Journal of Psychological Assessment, doi: 10.1027/1015-5759/a000272

Bach, B., Lockwood, J. \& Young, J. E. (2017). A new look at the schema therapy model: Organization and role of early maladaptive schemas. Cognitive Behaviour Therapy. doi: 10.1080/16506073.2017.1410566

Cid, J. (2010). Teràpia Esquemes. Actualitzacions de la Societat Catalana de Recerca i Teràpia del Comportament. CdScritc 2.0. Recuperado desde https://sites.google.com/site/actscritc/home/15-altres-arees/15-2

Cid, J., \& Torrubia, R. (2010). Adaptación española del Cuestionario de Esquemas de Young en la versión 3 reducida [Spanish version of the Young Schema Questionnaire-S3]. Manuscrito no publicado.

Cid, J. (2016). El esquema como organizador de la personalidad y sus trastornos: Estudio psicométrico de la adaptación española del Young
Schema Questionnaire-Short Form (Tesis doctoral). Universidad Autónoma de Barcelona. Recuperado desde https://www.tdx.cat/ handle/10803/370109

Calvete, E., Orue, I. \& González-Diez, Z. (2013). An examination of the structure and stability of early maladaptive schemas by means of the Young Schema Questionnaire-3. European Journal of Psychological Assessment, 29, 283-290.

Hair, J., Black, W., Babin, B. \& Anderson, R. (2009). Multivariate data analysis. Upper Saddle River, NJ: Prentice.

Hawke, L. D. \& Provencher, M. D. (2012). The Canadian French Young Schema Questionnaire: Confirmatory factor analysis and validation in clinical and nonclinical samples. Canadian Journal of Behavioural Science, 44, 40-49.

Hirsch, N., Hautekeete, M. \& Kochman, F. (2001). Early maladaptative processes, depression and alexithymia in suicidal hospitalized adolescents. L'Encephale, 21, 61-70.

Jacob, G. A. \& Arntz, A. (2013). Schema therapy for personality disorders: A review. International Journal of Cognitive Therapy, 6, 171-185.

Kriston, L., Schäfer, J., Jacob, G. A., Härter, M. \& Hölzel, L. P. (2013). Reliability and validity of the german version of the Young Schema Questionnaire - Short Form 3 (YSQ-S3). European Journal of Psychological Assessment, 29, 205-212

Lee, S. J., Choi, Y., Rim, H., Won, S. H. \& Lee, D. W. (2015). Reliability and validity of the korean Young Schema Questionnaire-Short Form-3 in medical students. Psychiatry Investigation. doi: 10.4306/ pi.2015.12.3.295

Lee, C. W., Taylor, G. \& Dunn, J. (1999). Factor structure of the Schema Questionnaire in a large clinical sample. Cognitive Therapy and Research, 23, $441-451$.

Lyrakos, D.G. (2014). The validity of Young Schema Questionnaire 3rd Version and the Schema Mode Inventory 2nd Version on the greek population. Psychology. doi: 10.4236/ psych.2014.5505

Masley, S.A., Gillanders, D.A., Simpson S. G. \& Taylor, M.A. (2011). A systematic review of the evidence base for schema therapy, Cognitive Behaviour Therapy, doi:10.1080/16506073.2011.614274

Mauchanda, P., Lachenal-Chevallet, K. \& Cottrauxc, J. (2011). Validation empirique du questionnaire abrégé des schémas de Young (YSQ-S2) sur une population de patients présentant un trouble de la personnalité borderline et de sujets témoins. L'Encéphale, 37, 138-143. doi: 10.1016/j.encep.2010.04.014

Oei, T. P. S. \& Baranoff, J. (2007). Young Schema Questionnaire: Review of psychometric and measurement issues. Australian Journal of Psychology, 59, 78-86.

Oettingen, J., Chodkiewicz, J., Mącik, D. \& Gruszczyńska, E. (2017). Polish adaptation of the Young Schema Questionnaire Short Form (YSQ-S3-PL). Psychiatria Polska, doi: https://doi.org/10.12740/PP/ OnlineFirst/76541

Rijo, D. \& Gouveia, P. (2008). A confirmatory factor analysis study of the YSQ-S3 in a large portuguese sample. Paper Presented at the 3rd Annual International Society of Schema Therapy Meeting, Coimbra, Portugal, October.

Riso, L. P., Froman, S. E., Raouf, M., Gable, P., Maddux, R. E., ... Turi- 
ni-Santorelli, N. (2006). The long-term stability of early maladaptive schemas. Cognitive Therapy and Research, 30,515-529.

Saariaho, T. Saariaho, A. Karila, I. \& Joukamaa, M. (2009). The psychometric properties of the Finnish Young Schema Questionnaire in chronic pain patients and a non-clinical sample. Journal of behavior Therapy and Experimental Psychiatry, 40, 158-168.

Sakulsriprasert, C., Phukao, D., Kanjanawong, S. \& Meemon, N. (2016). The reliability and factor structure of Thai Young Schema Questionnaire-Short Form 3. Asian Journal of Psychiatry, 24, 85-90.

Saritas, D. \& Gençoz, T. (2011) Psychometric properties of Young Schema Questionnaire- Short form 3 in a Turkish adolescent sample. Journal of Cognitive and Behavioral Psychotherapies, 9, 135-147.

Shorey, R. C., Anderson, S. E. \& Stuart, G. L. (2012). Gender differences in early maladaptive schemas in a treatment-seeking sample of alcoholdependent adults. Substance Use \& Misuse, 47, 108-116.

Shorey, R. C., Stuart, G. L. \& Anderson, S. (2012). The early maladaptive schemas of an opioid-dependent sample of treatment seeking young adults: A descriptive investigation. Journal of Substance Abuse Treatment, 42, 271-278.

Shah, R. \& Waller, G. (2000). Parental style and vulnerability to depression: The role of core beliefs. The Journal of Nervous \& Mental Disease, 188, 19-25.

Sines J., Waller G., Meyer C. \& Wigley L. (2008). Core beliefs and narcissistic characteristics among eating disordered and non-clinical women. Psychology \& Psychotherapy: Theory, Research y Practice, 81, 121-129.

Soygüt, G., Karaosmanoglu, A. \& Cakir, Z. (2009). Assessment of early maladaptive schemas: A psychometric study of the turkish Young Schema Questionnaire - Short Form-3. Turkish Journal of Psychiatry, 20, 1-10.

Thimm, J. C. (2011). Incremental validity of maladaptives schemas over five-factor model facets in the prediction of personality disorders symptoms. Personality and Individual differences, 50, 777-782.

Trip, S. (2006). The romanian version of Young Schema Questionnaire-Short Form 3 (YSQ-S3). Journal of Cognitive and Behavioral Psychotherapies, 6, 173-181.

Young, J. E. (1990). Cognitive therapy for personality disorders: A schema-focused approach. Sarasota, FL: Professional Resource Exchange.

Young, J. E. (1994). Cognitive therapy for personality disorders: A schema-focused approach, 2nd ed, Sarasota, FL: Professional Resource Exchange.

Young, J. E. (1999). Cognitive therapy for personality disorders: A schema-focused approach, 3rd ed, Sarasota, FL: Professional Resource Press.

Young, J. (2006). Young Schema Questionnaire-3. New York, NY: Cognitive Therapy Center.

Young, J. \& Klosko, J. S. (2007). Terapia de esquemas. En J. Oldham, A. Skodol y D. Bender (eds.), Tratado de los trastornos de la personalidad (pp. 289-322). Barcelona: Elsevier/Masson.

Young, J. Y., Klosko, J. S. \& Weishaar, M. E. (2003). Schema therapy: A practitioner's guide. New York: Guilford Press.

Waller, G., Shah, R., Ohanian, V. \& Elliott, P. (2001). Core beliefs in bulimia nervosa and depression: The discriminant validity of Young's Schema-Questionnaire. Behavior Therapy, 32, 139-153.

Welburn, K. R., Coristine, M., Dagg, P., Pontefract, A. \& Jordan, S. (2002). The Schema Questionnaire-Short Form: Factor analysis and relationship between schemas and symptoms. Cognitive Therapy y Research, 26, $519-530$. 